\title{
République Fédéral de l'Éthiopie : Vers une stabilité démocratique ou la dissolution?
}

\author{
Alex Héroux - Université de Concordia
}

\section{Introduction}

Il y a maintenant un peu plus de deux décennies que l'Éthiopie décida d'instaurer le système fédéralisme afin d'améliorer le processus démocratique ainsi que le niveau de vie pour l'ensemble du pays. De ce fait, le but de ce document est d'analyser les conséquences que cette décision a engendrées jusqu'à présent, pour ensuite examiner si cette fédération sera en mesure de perpétuer à travers le temps. En d'autres mots, nous allons observer si l'Éthiopie se dirige vers la stabilité ou l'échec. La nécessité de cette recherche ne vient pas seulement de la précarité politique à laquelle l'Éthiopie fait face, mais davantage des caractéristiques uniques qui constituent l'organisation de ce pays. Étant composés de plus quatre-vingts différents groupes ethniques, en plus d'une structure politique instable, les enjeux auxquelles ce pays fait face sont extrêmement complexes (Habtu 2004; Kincaid \& al. 2010). De plus, l'étonnant niveau de décentralisation offert par la constitution est si élevé que cela est dangereux pour la conservation du pays (Habtu 2005; Cohen 1995). Par ce fait même, afin de répondre à notre questionnement principal nous allons tenter de prouver notre hypothèse qui est la suivante : dû à l'organisation du pays sous une fédération du type "ethnique » ainsi que l'article 39 de la constitution, encourageant l'auto-détermination et autorisant la sécession, l'Éthiopie se dirige vers l'échec. Il ne fait donc aucun doute que sans changement considérable ce pays n'atteindra pas une stabilité désirée pour assurer un bon fonctionnement, ce qui mènera à sa dissolution.

La façon dont nous avons structuré le texte va nous permettre de bien comprendre le contexte éthiopien tout en défendant notre thèse de façon organisée. Nous allons débuter par expliquer le contexte historique de l'Éthiopie, puisqu'il est important de voir le rôle du passé et l'influence de celui-ci dans le fonctionnement de la présente fédération. Ensuite, nous allons enchainer avec l'argumentation de notre thèse qui sera divisée en trois sections. Les deux premières sections concernent respectivement les deux raisons que nous avons citées dans notre hypothèse, à savoir pourquoi le pays se dirige vers l'échec. La troisième section constitue les solutions que nous proposons pour la fédération. Nous terminons le document en concluant le tout.

\section{Contexte historique}

Étant considérés comme une nouvelle fédération, nous pouvons tout juste commencer à examiner l'efficacité et les répercussions que le système fédéralisme détient sur ce pays. La signature de la constitution en 1994, suivie de l'application de celle-ci en 1995 consiste au point 
départ de la fédération comme nous la connaissons aujourd'hui. Le but n'étant pas d'expliquer le parcours historique de l'Éthiopie, il est cependant nécessaire de brièvement notifier les évènements importants qui ont influencé le cheminement de la république fédérale. Dans cette ligne d'idée, nous allons alors brièvement relater les épisodes importants qui ont poussé l'Éthiopie à adopter un système fédéralisme. À la suite de cette description, nous pourrons nous concentrer sur le déroulement de la fédération depuis l'application de la constitution jusqu'à maintenant.

Lorsque nous observons attentivement la répression à laquelle les différents groupes éthiques ont fait face au cours du temps, il n'est pas étonnant que la constitution de 1994 mette l'emphase sur l'égalité de chaque ethnicité. Dès le début du 19e siècle, sous l'empereur Menilek II, l'objectif était de créer une nation Amharique constituée d'une ethnicité, d'une langue et d'une culture (Turton 2006, 119-123). La stratégie qui était de construire une nation c'est plutôt révélé en la destruction du pays. Par conséquent, divers mouvements se sont créées à la suite des années 1960 dont le Mouvement Étudiant Éthiopien (MÉÉ) qui était les premiers à réclamer l'égalité ethnique. Le mouvement de libération ethnique c'est poursuivi sous le groupe EPRDF (Ethiopian People Revolutionary Democratic Front), résultant à l'achèvement du régime dictatorial en 1991 (Turton 2006, 122-128; Tewfik 2010, 4-5). La révolution donna donc pour la première fois la chance à toutes les ethnicités du pays d'être à niveau égales et non dirigées par les amharas. Dès lors, l'Éthiopie entra dans une période de transition, de 1991 à 1995, où les élites étaient à la recherche d'un système pouvant améliorer à la fois les relations entres différentes ethnicités dans le pays et la stabilité du système politique. De là proviennent le système fédéralisme et l'Éthiopie contemporaine.

L'application de la constitution en 1995 a provoqué de nombreux changements dans la manière dont le pays fonctionne et il sera à nous de juger si ces modifications sont pour le mieux du pays. D’abord, le pays a été séparé en 9 régions et 2 villes détenant un statut spécial, Addis Ababa qui est la capitale fédérale et Dire Dawa la capitale territoriale (voir Annexe1, carte 1.0). De plus, chacune de ces régions est séparée par des zones administratives et des zones spéciales nommées wereda. La particularité de l'Éthiopie est que les 9 régions ont été formées en lien avec les différentes ethnies, de là vient l'appellation « fédération ethnique ». Les motifs derrière ce type de séparation sont bien évidemment, éviter les conflits raciaux datant de longue date. D'ailleurs, à l'exception des élites, les interactions entre les différentes régions sont limitées, puisque la langue la plus parlée (l'amharique) est seulement utilisée par un peu plus de $30 \%$ de la population (Habtu $2004,105)$. Il y a donc une panoplie de langues dans ce pays faisant provoquant un faible niveau de communication à l'intérieur du pays. Néanmoins, depuis 1995, il est légitime d'affirmer que la situation économique ainsi que le niveau de vie, encore plus dans les régions, s'est grandement améliorée. Grâce aux standards nationaux que les régions doivent maintenant respecter, entre autres en éducation et santé, l'ensemble de la société bénéficie de meilleures conditions (Tewfik 2010, 16). 
Ensuite, ne voulant pas revivre les incidents du passé, la constitution a été écrite de façon à n'entraver aucun droit humain et de laisser la chance à chacune des ethnies de s'émanciper. La diversité est mise de l'avant à un point tel que dans le préambule de la constitution nous pouvons lire "We the Nations, Nationalities and People of Ethiopia », ceci reflète bien l'optique vers lequel le pays veut se diriger (Turton 2006, 132). En outre, la constitution intègre des clauses pour les régions, telles que le droit de sécession et d'auto-détermination, le droit d'écrire leur propre constitution, le droit de choisir leur langage officiel, le droit d'établir leurs propres institutions et le droit d'avoir leur propre force policière (Cohen 1995, 165). Avec des conditions de la sorte, il ne fait aucun doute que l'Éthiopie se retrouve du coté de l'extrême décentralisation si on s'en remet au spectrum de Friedrich (Burgess 2006, 35). En mettant l'emphase sur l'ouverture et l'accommodation, la constitution éthiopienne encourage la flexibilité et l'autonomie des régions. Il est de mise qu'en optant pour une fédération ethnique, les élites devaient accepter le fait de donner une grande partie des pouvoirs aux régions pour que celles-ci se développent en respect avec leurs identités. Toutefois, la situation à laquelle nous avons assisté jusqu'à présent ne se déroule pas parfaitement comme la constitution le désire. C'est-à-dire, le gouvernement national exerce un énorme pouvoir sur les gouvernements régionaux et l'ensemble du pays de sorte que les droits constitutionnels ne sont pas respectés comme il le devrait (Kincaid 2010,159-160; Turton 2006, 156-157). De ce fait, malgré l'amélioration croissante du niveau de vie, l'instabilité politique persiste dans le pays. Cette instabilité qui a des répercussions partout au pays vient essentiellement du manque de démocratie dans le processus politique. Il est donc évident que la fédération éthiopienne est encore en développement. Or, l'histoire de ce territoire est très longue, beaucoup de conflits et d'évènement marquant reste gravé dans la mémoire des différentes ethnies, c'est pour cette raison que cette nouvelle fédération est aussi fragile.

Maintenant que nous avons établi une fondation abrégée de la situation éthiopienne, nous sommes prêts à nous diriger vers la section principale du texte. Ayant déjà expliqué le contexte éthiopien, la section suivante sera consacrée exclusivement à prouver notre thèse. Dans ces conditions, notre opinion poussera la réflexion à savoir si les faits exposés plus haut reflètent réellement une un futur positif pour l'Éthiopie ou non.

\section{Une fédération perdue}

Selon la description que nous avons établie ultérieurement de la fédération éthiopienne, il serait compréhensible d'affirmer que l'instauration du fédéralisme a réformé le fonctionnement du pays pour le mieux. Or, comme nous avons mentionné dans notre introduction, nous pensons que l'Éthiopie se dirige vers un désastre. Cette partie du texte est donc dédiée à notre argumentaire afin de défendre notre hypothèse de départ. Nous avons basé notre hypothèse sur deux raisons assez claires : les répercussions du fédéralisme du type « ethnique » et les conséquences de la clause de sécession dans le contexte éthiopien. De cette manière, nous exposerons le futur incertain de 
l'Éthiopie dans ces deux sections, pour finalement illustrer des solutions qui pourraient améliorer l'avenir de cette nouvelle fédération.

\section{Fédéralisme du type ethnique}

Le fédéralisme ethnique était perçu comme la meilleure solution afin d'éviter les conflits ethniques interminables dans ce pays, or, avec du recul, il est discutable à savoir si cela était vraiment une bonne décision. En fait, ce type de fédéralisme a apporté plusieurs nouveaux problèmes, en débutant par la séparation des régions. Dans un pays comme l'Éthiopie, il est très difficile d'attribuer une région à une ethnie. D'ailleurs, pendant la période de transition le pays était divisé en 13 régions plus les deux villes (voir Annexe 1, Carte 1.1). Cela signifie que non seulement des bordures ont été créées pour la période de transition, mais qu'après celle-ci, nous en avons créée de nouvelles. Déjà que la modification de territoire est une opération délicate, surtout lorsqu'il y a de fortes tensions ethniques, les modifier à deux reprises en moins de 5 ans est très risquée. Évidemment, l'élimination de certaines régions après 1995 a créé de nouveaux conflits entre différentes ethnies. L'application du fédéralisme, établi pour stabiliser le pays, à donc dès le départ produit l'émergence de nouveaux conflits. Il est donc difficile d'argumenter que cela a amélioré la paix dans le pays, il serait plus juste de dire que cela à rediriger les problèmes.

Toutefois, ces problèmes ethniques sont peu surprenants et risques de refaire surface sans arrêt dû à la définition du mot « ethnie » que le pays utilise. L'Éthiopie définit un groupe ethnique par rapport à sa langue, ce qui est tout à fait légitime pour la majorité des pays, mais lorsqu'il y a plus de 80 langues parlées il est impossible de tous les accommodé (Turton 2006,134). La situation est encore plus importante ici où les deux langues les plus parlées dans le pays sont seulement parlées par un peu plus de 30\%, la troisième langue est parlée par $6.07 \%$ de la population (voir Annexe 2, tableau 2.0). Pourtant, si on se rappelle bien le préambule de la constitution spécifie bien : «We the Nations, Nationalities and People of Ethiopia». Ainsi, en tentant d'accommoder toutes ethnies/langues, le fédéralisme ethnique produit littéralement de nouveaux conflits et des enjeux extrêmement complexes. Aussi bien que, la source de ces nouveaux conflits est très facile à cibler, puisque pratiquement chaque région est constituée d'une majorité ethnique et de minorités ethniques. Conséquemment, comme les régions ont le droit de choisir la langue qu'ils désirent, il revient à dire que le groupe ethnique majoritaire impose sa propre langue pour l'entièreté de la région. C'est pourquoi nous pensons que d'avoir implanté un système fédéralisme ethnique donne l'impression d'avoir apporté une équité entre les différentes ethnies du pays. Cependant, le problème a seulement été transféré du niveau national au milieu régional, ce qui fait en sorte qu'il soit moins apparent. Partout où il y aura une majorité, il y aura une minorité. Ce qui est dangereux est que contrairement à avant où les conflits se déroulaient entre l'entièreté de la population contre le gouvernement national, les conflits se déroulent maintenant entre la population même. Du coup, la répression est difficilement contrôlable, surtout dû au fait que les régions détiennent leur propre force de l'ordre. Donner plus d'autonomie aux régions n'a pas eu le résultat attendu. Au lieu d'avoir une répression ethnique dans l'ensemble du pays, l'Éthiopie témoigne présentement d'intimidation 
dans chacune des différentes régions. Alors, le problème ethnique était l'une des principales raisons pourquoi le fédéralisme ethnique a été adopté, mais il ne semble pas avoir de progrès à ce niveau. L'élimination de problème en a seulement apporté de nouveaux.

Jusqu'ici nous avons vu que l'organisation du territoire selon les ethnies n'a pas été aussi fructueuse qu'espéré. La déception de l'organisation territoriale ne fait qu'augmenter lorsque nous analysons ce que les dirigeants ont décidé de faire avec la région du SNNP (Southern Nations, Nationalities and People Region). Généralement, les régions contiennent entre 1 et 5 différentes ethnies afin de tenter de conserver un bon équilibre, or, la région du SNNP contient 46 différentes ethnies (voir Annexe 2, Tableau 2.1). En d'autres mots, cette région a été établie pour y installer toutes les ethnies qui n'étaient pas conformes aux autres régions. Les problèmes qu'attend cette région sont énormes. Aussi bien que ceux-ci ont déjà débuté, prenons le cas de la communauté Silte dans la région du SNNP qui au début des années 2000 s'est séparée de la communauté Gurage à laquelle elle était identifiée, car elle se disait distincte de celle-ci. Par conséquent, après avoir fait un référendum chez les Siltes ceux-ci se sont séparés et ont maintenant leurs propres communautés (Habtu 2004, 108-109). Les problèmes émergeants du fédéralisme ethnique ne sont pas seulement en matière de guerres, mais aussi en terme de séparations de populations. La flexibilité et la facilité de se faire reconnaitre comme une ethnie distincte sont trop élevées, ce processus peut être utilisée avec abus auprès des différentes ethnies.

Cette situation peu semblée peu importante, mais en ayant une communauté distincte les groupes ethniques reçoivent plus d'argent du gouvernement national pour développer leurs propres institutions. C'est à ce moment que l'importance de cet enjeu prend tout son sens, les différentes ethnies sont plus conscientes de la facilité de ce processus et peuvent agir en conséquence. Le fédéralisme ethnique encourage donc les différences entre ethnies qui n'étaient pas réellement présentent avant l'instauration de ce système. Les répercussions de ce système peuvent être sans fin, puisqu'il a plus de 80 langues parlées. Pourtant, lorsque nous prenons le temps d'analyser les différentes ethnies, nous en venons à constater que la majorité de celles-ci sont mélangées entre elles et que la raison d'être du fédéralisme ethnique perd tout son sens. La séparation de la population a été jugée plus importante que l'unification de celle-ci, ce qui met certainement à risque l'entité du pays.

À ce moment-ci, il est tout à fait légitime de se demander si l'application du fédéralisme ethnique a vraiment augmenté le niveau d'accommodation ou bien si celui-ci a simplement apporté de nouveaux obstacles au développement du pays. Il y a eu tellement d'efforts qui ont été consacrés à résoudre les conflits ethniques que les autres secteurs en ont soufferts. Tel est le cas pour les institutions et les infrastructures qui ont certainement été négligées aux dépens de la séparation ethnique. Au lieu de tenter de garder tous les groupes ensembles en leur permettant de pouvoir s'identifier à leur ethnie facilement, il aurait été une meilleure idée de réunir les différentes populations sous un même projet, comme les institutions et les infrastructures sont capables de le 
faire. Ce que nous voulons dire est qu'en décentralisant le plus de pouvoir possible, l'importance du gouvernement national au niveau de la population est plus ou moins élevée. C'est situation s'avère véridique dans le cas où les institutions fédérales sont quasi-invisibles dans les différentes régions de l'Éthiopie, ce qui cause des limites sérieuses au projet national (Kincaid 2010,151). Bien que tenter d'accommoder et d'intégrer le plus possible toutes les populations en leur accordant de l'autonomie soit bien, et même encourager, si cela n'est pas soutenu par un mouvement d'unification, ce projet est dédié à l'échec (Idem, 152).

Dans les pages précédentes nous avons étudié les conséquences non anticipées par les dirigeants qui ont décidé d'installer un fédéralisme ethnique en Éthiopie. Ce qui est le plus effrayant en regardant ces conséquences est que celles-ci s'entrecroisent constamment. En d'autres termes, en observant tous ces conséquences en même temps, il est raisonnable d'anticiper un renforcement de division entre les régions et les différentes ethnies. Cette particularité de l'Éthiopie est très dangereuse, c'est-à-dire, non seulement la fédération présente des renforcements de division entre les régions, mais aussi à l'intérieur même des régions. Il est facile de comprendre le renforcement entre régions, puisque nous pouvons comparer la situation avec la première fédération africaine, le Nigéria, qui a aussi beaucoup d'ethnies. Dans ses 3 périodes démocratiques, séparées par des régimes militaires, le Nigeria a débuté avec 3 états (1960), pour ensuite augmenter à 19 états (1979) et finalement à 36 états (1999) (Turton 2006, 68). En analysant l'évolution du Nigeria nous percevons que quand il y avait peu d'états et que ceux-ci étaient grands, il y a avait de forts mouvements de renforcements de séparations. Ce qui nous laisse croire que plus les états sont puissants, plus le renforcement est élevée. En prenant ces informations pour l'Éthiopie, en plus de l'absence d'institutions fédérales dans les régions, tous les ingrédients y sont pour l'accroissement du renforcement de divisions. En plus, depuis 1999 le Nigéria a adopté une philosophie axée sur le partage fédéral et régional pour assurer un mouvement d'unification et un degré d'autonomie aux états, ce qui n'est pas le cas pour l'Éthiopie (Idem, 72).

La comparaison de l'Éthiopie avec l'évolution du Nigéria illustre parfaitement les dangers vers lesquelles l'Éthiopie se dirige, si aucun changement n'est fait. Contrairement au Nigéria le partage est absent de la structure éthiopienne, tout est concentré sur l'auto-détermination. À un point tel que les différentes communautés tentent de se faire reconnaitre comme une communauté distincte afin de contrôler l'argent pour développer leurs projets. Ceci est sans compter qu'il n'y a aucun symbole encourageant l'unification du pays ou auquel la population peut s'identifier. L'unique symbole éthiopien qui pourrait être considéré est que le pays n'a jamais été colonisé. Or, celui-ci n'est pas mis à l'avant-plan dans le pays et est plutôt faible. En ce qui concerne le renforcement de division à l'intérieur même des régions, ceci est directement relié au point que nous avons fait auparavant en lien avec les minorités qui sont contrôlées les majorités ethniques dans une région. D'autre part, si nous regardons la région du SNNP qui est constituée de minorités, ceux-ci préfèrent clairement vivre indépendamment que de former de plus larges collectivités. Ceci 
est facilement observable, car c'est dans cette région que nous pouvons retrouver le plus de zones administratives en Éthiopie.

Ainsi, il est important de mentionner que nous ne s'opposons pas aux instruments d'intégrations utilisées par les fédérations. Au contraire, il faut assurer la facilité d'intégration afin que le fonctionnement de la société soit efficace. Toutefois, le problème ici est que l'Éthiopie n'a pas utilisé correctement ces instruments. La maladresse du fédéralisme ethnique peut à elle seule faire effondrer la fédération, car la séparation est mise en avant plan. Bien qu'il soit important d'offrir un niveau d'autonomie aux régions, celles-ci doivent partager un sentiment d'appartenance avec le pays. Dans ce cas-ci, non seulement les régions ne soutiennent pas l'unification du pays, mais elles tentent d'acquérir toujours plus d'autonomie afin de s'auto-diriger. En retour, ce comportement n'est pas unique à l'Éthiopie, dans toutes les fédérations certaines régions tentent d'être plus autonome pour mieux servir leur population. Cependant, ce qui est unique à l'Éthiopie est que la façon dont le fédéralisme ethnique est développé leur donne pratiquement carte blanche et encourage cette séparation.

\section{Clause de sécession}

Ce qui distingue le plus l'Éthiopie des autres systèmes fédéraux, est sans aucun doute la clause de sécession dans la constitution (voir annexe 3, article 39). La controverse de cette clause nous oblige à nous interroger à savoir si le pays ne se situe pas plus près de la confédération que de la fédération, dû au niveau élevé d'indépendance des régions. Néanmoins, en analysant la formation du pays, il est clair que cette clause était une condition nécessaire afin d'obtenir la signature de toutes les régions, surtout la région du Somali (Habtu 204, 324-325). Malgré les quelques conditions à respecter pour se séparer; un vote des plus des deux-tiers des membres du conseil législatif de la région approuvant la demande et un vote référendaire organisé par le gouvernement national, nous pensons que cette clause est bien plus que seulement symbolique. Certains experts de l'Éthiopie, dont Habtu, affirment que cette clause est purement symbolique. Or, la clause aurait été seulement symbolique si le gouvernement national avait un impact et une visibilité constante dans la vie des citoyens. Dans le cas présent la population est reliée presque uniquement à sa région, ce qui réduit l'importance du gouvernement fédéral à leurs yeux et renforcie l'importance de la clause de sécession.

En outre, l'importance de cette clause pour certains groupes ethniques était primordiale due à la répression qui a eu lieu pendant plusieurs années. De ce fait, puisque la répression ethnique par le gouvernement national est maintenant absente depuis de nombreuses années il peut sembler impensable qu'une région se sépare sans l'apparition d'un conflit. En revanche, nous pensons plutôt le contraire, à savoir que le développement des régions et leurs autonomies les encouragent à se développer encore plus, mais de façon indépendante. Ce que nous voulons dire est que les probabilités que les régions veuillent se séparer avant que la fédération soit établie étaient faibles dus au très bas niveau de vie. Maintenant, grâce à la fédération nous ne pouvons nier que le niveau de vie à raisonnablement augmenter, ce qui signifie une meilleure économie et des populations plus 
éduquées. Par conséquent, étant plus conscient des enjeux et étant conscient de leurs potentiels, la tentation de se séparer et de former leur propre nation peut sembler plus attrayante et surtout plus réalisable. Ainsi, cette clause n'est plus simplement symbolique comme elle pouvait l'être auparavant, mais bien une option envisageable.

D’ailleurs, la fédération éthiopienne a déjà vécu une séparation lors de ses débuts avec ce qui est maintenant le pays de l'Érythrée. Bien que le contexte soit différent, l'inspiration et le fait que la séparation soit effectivement une option envisageable à assurément un impact sur la conscience des populations. En fait, l'ex-région de l'Érythrée avait des raisons plus que valables de désirer un pays indépendant, dû à la répression que la population subissait et au régime autoritaire qui régnait. Cependant, tout comme l'Érythrée, les courantes régions ont plus de raisons que la constitution peut le laisser croire de se séparer, puisque en réalité l'Éthiopie n'est pas un pays entièrement démocratique. Nous considérons l'Éthiopie comme un pays semi-autoritaire. Cette situation apporte une toute nouvelle dynamique à la clause de sécession, puisque les droits de la population ne sont pas tous acceptés. Si la fédération était véritablement un pays démocratique comme elle prétend l'être, les régions seraient moins en mesure de vouloir se séparer car tous leurs droits seraient respectées. Or, la population éthiopienne n'a pas cette chance. L'exemple le plus explicite de ce régime semi-autoritaire est lorsque nous analysons les élections. Depuis 1995, quatre élections ont eu lieu soit en 1995, 2000, 2005 et 2010. Les deux premières élections ont été remportée par l'EPRDF (Ethiopian People's Revolutionary Democratic Front), mais il n'avait aucun opposant. En retour, dans les deux dernières élections plusieurs parties légitimes faisaient la course. Cependant, l'EPRDF qui a encore gagné a été accusé d'intimidation et de violence afin de s'assurer de remporter les élections (aleen 2006, 250-251).

Les conséquences de ce système semi-autoritaire sont très importantes pour la fédération et l'utilisation de la clause de sécession. Les régions ont accepté de faire partie de cette entente de fédération afin de faire partie d'un système auquel ils pouvaient avoir confiance et où leurs intérêts seraient représentés. Par ce fait même, l'emprise du parti EPRDF ne peut qu'apporter du mécontentement auprès des différentes régions qui seraient mieux représentées avec un autre parti au pouvoir. En observant le déroulement de la séparation de l'Érythrée, où la séparation a été très violente, il est légitime de craindre un scénario similaire pour les années futures. Quoique le contexte soit différent, la démocratie n'est toujours pas présente et les droits constitutionnels ne sont pas tous respectés. Dès lors, lorsque nous analysons les pré-conditions d'une fédération échouée, le non-respect des règles constitutionnelles est l'une des causes principales. Alors, le fait que l'Éthiopie soit dirigée par un parti semi-autoritaire, où les droits humains ne sont pas tous respectés, oblige les régions désavantagées par cette situation à penser à utiliser la clause $39 \mathrm{si}$ aucune correction n'est faite.

D’un point de vue opposé, il est facile de penser que puisque les régions sont sous un régime semi-autoritaire, jamais leur droit de sécession ne sera respecté. Ce qui est effectivement un 
scénario très plausible. Cependant, la particularité de la clause 39 est qu'elle forme la clause la plus importante de la constitution. C'est-à-dire qu'elle est la seule clause qui doit être appliquée à tout moment, même lorsque le pays est en « état d'urgence ». Conséquemment, si cette clause n'est pas respectée, nous pensons fortement que le pays assistera à une guerre civile ou de sérieux conflits, due à l'importance de cette clause aux yeux des éthiopiens. Ainsi, l'impact du régime autoritaire sur la clause 39 apportera soit une région à se séparer grâce à cette même clause où apportera le pays en guerre, car la clause de sécession ne sera pas respectée par le gouvernement.

Dans la même ligne d'idée, depuis la création de la fédération, comme nous l'avons mentionné, le pays a seulement été dirigé par l'EPRDF. Ce qui nous obliges à nous interroger à savoir si la transition d'un nouveau parti serait sans dommage pour le pays. Étant une fédération du type ethnique, il est logique de voir pratiquement que des partis représentant une certaine région ou ethnie. L'impossibilité de représenter tous les groupes du pays est un enjeu crucial pour le futur de la fédération, surtout si nous assistons à l'émergence au pouvoir de partis voulant seulement avantager leur région. Curieusement, nous pouvons comparer la situation de l'Éthiopie avec l'ExYougoslavie et l'URSS. C'est deux pays utilisant le système fédéralisme étaient maintenus en vie grâce à un groupe de dirigeant, ou un seul, dirigeant rassemblant la nation. Lorsqu'il y a un changement de gouvernement dans ces deux pays, plusieurs conflits ont fait surface et la guerre a éclaté. Ces exemples nous obligent à considérer ce scénario pour l'Éthiopie. De plus, ces deux exfédérations avec chacune clause de sécession à l'intérieur de leur constitution, ce qui laisse un mauvais présage dû au fait que se sont les seuls fédérations avec l'Éthiopie à avoir une telle clause . Les ressemblances sont étrangement intéressantes, la seule distinction, qui est très importante, est que les deux anciennes fédérations prônaient une seule ethnie, culture et langage à l'intérieur du pays.

Or, reste que le mode de gouvernance autoritaire et même violent utilisé par l'EPRDF est inquiétant pour le futur de l'Éthiopie. Même si la répression ethnique n'est plus pratiquée, nous avons deux exemples de fédérations utilisant la violence et ayant un système autoritaire, qui ont tous deux terminés en catastrophe. Ces exemples renforcissent notre idée que si une région demande la clause de sécession, le pays se transforme en état de guerre. Nous soutenons ces opinions par le fait que les fédérations non démocratiques précédentes qui n'ont pas respecté la constitution de façon adéquate (Ex-Yougoslavie et URSS) ont connu des guerres sanglantes. Alors, initialement nous trouvions la clause de sécession dangereuse dans un pays aussi décentralisé que l'Éthiopie, mais maintenant cette clause est encore plus dangereuse puisqu'elle est présente dans un pays semi-autoritaire. En outre, même si nous avons vu que le gouvernement accepte toutes les ethnies, cultures et langues, il reste que celui-ci n'accepte pas les idéologies différentes à un point où il utilise la violence. Ainsi, sous une forme de répression différente que les deux ex-fédérations, le cas de l'Éthiopie n'est pas à l'abri d'une catastrophe. 
D'ailleurs, ce qui est encore plus inquiétant est que le parti au pouvoir ne respecte déjà pas une clause majeure de la constitution, qui est la représentation de chaque ethnie dans la chambre de la fédération. La chambre de fédération est sans doute un des instituts les plus importants pour la fédération, car elle assure une représentation proportionnelle des différentes ethnies dans le pays. Selon l'article 61 (voir annexe 3) de la constitution, chacune des ethnicités doit être représentée par au moins un membre à la chambre de la fédération. Toutefois, cette clause qui est extrêmement importante pour le pays, puisqu'elle est l'une des raisons principales à savoir pourquoi les régions ont accepté de former une fédération, n'est pas respectée. Encore une fois l'effet de cette décision prouve non seulement que le respect de la constitution par le gouvernement est très faible, mais aussi que le " fédéralisme ethnique " perd tout son sens. La relation entre la clause 61 et la clause 39 de la constitution est intimement liée, car si la représentation est respectée les ethnies ont moins de chances de vouloir se séparer. Ce qui est encore plus étonnant est que ce n'est pas les ethnies les moins nombreuses qui ne sont pas représentées. En fait, l'ethnie la moins nombreuse de l'Éthiopie avec environ 1500 personnes est représentée à la chambre (Habtu 2005, 330). Ce qui signifie clairement un traitement inégal des différentes ethnies dans les institutions politiques. La contradiction entre le comportement du gouvernement et la constitution, comme nous l'avons mentionné ultérieurement, est l'un des critères principaux pour l'échec d'une fédération. Ce qui est encore plus grave ici, c'est que la chambre de la fédération est l'essence même de la représentation et de la preuve de l'accord fédéral entre les régions.

Après toutes les justifications exposées plus haut prouvant que la clause de sécession dans la constitution est dangereuse, et ce, surtout dans le contexte de l'Éthiopie, nous devons absolument examiner si des mouvements séparatistes sont en œuvres qui menaceraient la survie du pays. En fait, plusieurs partis séparatistes existent dont OLF (Oromo Liberation Front), ALF (Afar Liberation Front) et plusieurs autres partis moins importants. Le parti séparatiste le plus important n'est nul doute OLF, puisque les Oromos constituent la plus grande partie de la population éthiopienne. Or, il n'est pas étonnant de découvrir que le parti au pouvoir emprisonne les partisans et les dirigeants de ce parti séparatiste. L'absence de liberté d'expression et d'opinion due à la suppression peut qu'augmenter dans ce type de situation. Plus les gens verront leurs droits mis de coté et les gens qui les représentent emprisonnés, plus ils seront mécontents. Empêcher l'émergence de partis séparatistes, dont certains ont une popularité considérable, ne fera qu'augmenter le problème. Donc, oui les mouvements séparatistes existent, mais ceux-ci doivent se faire discret dû à la violence.

Ainsi, ce que nous avons voulu démontrer dans cette section du texte est que la clause de sécession dans la constitution semble reconnaitre et encourager l'émancipation des différentes ethnies. Cependant, la réalité est totalement différente. D'une part, cette clause met la fédération en danger en laissant croire que cette option est viable, et donc, les régions prennent en considération la sécession. De l'autre part, la façon dont le pays fonctionne contredit cette clause, ce qui provoque une insatisfaction grandissante chez les ethnies et les régions. De plus, en ayant brièvement 
comparé la situation avec l'Érythrée, l'Ex-Yougoslavie et l'URSS, où chacune des séparations s'est terminées en conflit dû au non-respect de la constitution, il est légitime d'anticiper le pire pour l'Éthiopie. La fragilité de l'Éthiopie a obligé les dirigeants à intégrer cette clause à la constitution, mais celle-ci pourrait bien être la raison de l'échec de la nouvelle fédération.

\section{Le futur de la fédération éthiopienne}

L'avenir de la République Fédérale éthiopienne est très incertain, mais il n'est toujours pas trop tard pour améliorer ses chances de survie. Nous avons vu au début du document que le niveau de vie de la population a progressé, surtout en ce qui concerne la santé et l'éducation. Or, le problème se retrouve dans l'organisation de la fédération et dans l'injustice qui y persiste. Comme nous l'avons illustré dans la section précédente, si rien n'est fait l'Éthiopie risque de grand danger. Bien que certains instruments d'intégrations aient été utilisés, ceux-ci requièrent certaines modifications pour les adapter au contexte éthiopien. D'ailleurs, le premier problème auquel nous devons nous attardés est le fédéralisme ethnique. Si l'idée de base était bonne, l'application de ce type de fédéralisme n'est pas efficace. Ce fédéralisme ramène l'Éthiopie sous un état tribal où tous les groupes sont séparés et se concentrent seulement sur leurs propres survies (Mahretu 2012, 119). En ce sens, l'Éthiopie doit absolument faire augmenter l'importance du niveau national et de montrer qu'il est possible de former un pays uni avec une multitude d'ethnies à travers le territoire. L'implantation de projet partagé ou de législation partagée entre le niveau fédéral et le niveau régional est sans aucun doute une solution envisageable et efficace. Cette solution s'est entre autres montrée performante dans le cas du Nigeria, où la collaboration a été mise de l'avant. L'équilibre entre l'autonomie des régions, la participation et contribution du niveau national est extrêmement bien exécuté depuis quelques années au Nigeria et cela se fait voir dans toutes les sphères du pays. Le Nigéria est sans doute l'un des pays les plus stables d'Afrique. L'Éthiopie devrait donc se diriger vers un fédéralisme collaboratif, ce qui permettrait au pays de se développer plus rapidement et efficacement sous une seule entité. En outre, en mettant l'emphase sur la collaboration plutôt que la séparation ethnique, les renforcements de divisions entre les régions vont grandement diminuer. De plus, dans un pays aussi fragile que l'Éthiopie en ce moment, celui-ci ne peut pas se permettre de vivre sous la présence des anciens conflits. Surtout que la séparation ethnique ne fait que conserver ces conflits en vie. Pourtant les ethnies se mixtes de plus en plus entre elles, ce qui enlève toute la nécessité de la séparation ethnique. La modernisation du modèle fédéralisme doit donc être prioritaire.

Dans une autre ligne d'idée, non seulement le gouvernement national doit développer plus d'institution dans les régions, mais il doit débuter par bien utiliser ceux qui sont déjà en place. Ce que nous voulons dire ici, est que la Chambre de la Fédération, qui représente un symbole de l'unification des régions, est trop peu utilisée (Kincaid 2010,151-152). Les membres de la deuxième chambre, se réunissent seulement à deux reprises à chaque année, ce qui est loin d'être suffisant pour assurer une gouvernance intègre (Idem, 146-147). À l'avenir, cette chambre assurant le respect de la constitution devrait être plus active au sein du pays et évidemment représenter 
chaque ethnicité, ce qui n'est toujours pas le cas, mais pourtant clairement identifié dans la constitution. Également, il pourrait être une bonne idée d'élire les représentants par un vote de la population. Présentement, les membres sont choisis par les délégués législatifs. D’ailleurs, en votant pour les membres de la Chambre de la fédération, la population s'acclimaterait avec l'action d'aller voter, ce qui peut certainement améliorer le processus lors des élections fédérales.

Bien entendu, le changement de direction ne sera pas facile à établir si le pays est toujours contrôlé par un gouvernement semi-autoritaire. Les solutions concernant cet enjeu sont extrêmement difficiles à implanter, puisque le pouvoir réside qu'a un seul endroit. Le moyen le plus efficace et qui réduit les chances de conflits est l'émergence de fortes oppositions politiques (Idem, 159-160). Si des partis d'opposition se forment et constituent un groupe légitime, la population voudra le changement et le parti qui est au pouvoir n'aura pas d'autre choix que d'ouvrir la discussion. En outre, si l'opposition pouvait établir une coalition afin de réunir les forces contre l'autorité, cela augmenterait grandement les chances de vaincre le régime. En d'autres mots ce qu'il faut est un mouvement solidaire de la part de la population est des groupes politiques afin de compléter la transformation de l'Éthiopie et d'assurer un avenir meilleur.

\section{Conclusion}

Pour conclure, nous maintenons notre thèse de départ disant que l'avenir de l'Éthiopie est incertain. Le fédéralisme du type ethnique et la clause de sécession sont tous les deux des instruments d'intégrations très délicats, car il est difficile de juger si cela apportera la stabilité ou l'échec du pays. Toutefois, dans le cas de l'Éthiopie il est plus logique que ces instruments tournent à l'échec, puisque le pays est trop instable. D'une part, l'application du fédéralisme ethnique dans un pays aussi instable que l'Éthiopie ne peut qu'accentuer la division entre les différentes ethnies. D'ailleurs, ce type de fédéralisme ramène le pays dans un état tribal, et donc empêche la formation d'un projet national. Sous une telle organisation où les différences ethniques sont à l'avant-plan, il est impossible de créer un pays uni et fière. En outre, si un pays décide d'utiliser le fédéralisme ethnique comme instrument d'intégration, celui-ci doit assurer une participation continuelle du niveau national. Si le gouvernement fédéral est trop absent dans la vie de la population, celui-ci devient inutile aux yeux de la société. L'apport de législation partagé où les deux niveaux de gouvernement participent est sans doute le moyen de pratiquer un fédéralisme ethnique tout en conservant une vision nationale. En ce qui concerne la clause 39, nous avons vu que celle-ci faisait partie de la négociation constitutionnelle et qu'elle était donc nécessaire pour que les régions intègrent la fédération. Or, encore une fois, cet instrument d'intégration est extrêmement puissant, mais aussi dangereux. Le fait que cette clause est intégrée dans un pays instable et dans une fédération qui est dirigé par un gouvernement semi-autoritaire nous oblige à anticiper le pire. En laissant croire aux régions qu'ils ont la possibilité de s'émanciper à l'extérieur de la fédération éthiopienne, la constitution semble très incitante. Cependant, la contradiction entre ce que la constitution affirme et ce que le gouvernement fait risque d'augmenter le niveau d'opposition et d'insatisfaction envers ce même gouvernement. De cette situation émerge la possibilité des régions 
de vouloir se séparer. Ce qui est encore plus dangereux avec cette clause, est que nous ne savons pas ce qui va se produire si une région l'utilise. C'est-à-dire, est ce que le gouvernement national va accepter et la région va se séparer ou le gouvernement national n'acceptera pas et une guerre jaillira dans ce pays instable. Cette clause apporte donc beaucoup de questions et mais surtout de l'inquiétude pour l'avenir de l'Éthiopie.

D'autre part, nous espérons évidemment que l'Éthiopie procèdera à des modifications afin de stabiliser le pays et d'assurer la continuité de cette récente fédération. L'enjeu fédéral de l'Éthiopie est extrêmement important pour le fédéralisme. En adoptant des instruments d'intégrations uniques, ce pays peut apporter une nouvelle vision de l'organisation fédérale surtout dans un monde où la diversité culturelle ne fait qu'augmenter à travers la planète. Si l'Éthiopie trouve un moyen d'adapter les failles qui sont présentes pour faire de ce pays un territoire stable, cela incitera assurément certains pays instables à tenter l'expérience fédérale. Ainsi, bien que l'avenir de l'Éthiopie est plus qu'incertain, il n'est pas trop tard pour trouver des solutions plus adaptés au contexte de ce pays et par le fait même d'inciter de nouveaux pays à utiliser le système fédéral pour accommoder leur diversité. 


\section{Bibliographie}

Aalen, Lovise. 2006. "Ethnic Federalism and Self-Determination for Nationalities in a SemiAuthoritarian State: The Case of Ethiopia." International Journal of Minority and Group Rights 13: 243-261.

Burgess, Michael. 2006. Comparative Federalism :Theory and Practice. London; New York, NY: Routledge.

Cohen, John M. 1995. ""Ethnic Federalism" in Ethiopia." Michigan State University Press 2 (2): 157-188.

Habtu, Alem. 2003. "Ethnic Federalism in Ethiopia: Background, Present Conditions and Future Prospects."Queen College, City University of New York.

- - - 2004. "Ethnic Pluralism as an Organizing Principle of the Ethiopian Federation." Springer 28 (2): 91-123.

- - 2 2005. "Multiethnic Federalism in Ethiopia: A Study of the Secession Clause in the Constitution." Oxford University Press 35 (2): 313-335.

Kavalski, Emilian and Magdalena Zólkos. 2008. Defunct Federalisms :Critical Perspectives on Federal Failure. Federalism Studies. Aldershot, Hampshire, England; Burlington, VT: Ashgate.

Kincaid, John, Luis Moreno, César Colino, International Association of Centers for Federal Studies, and Forum of Federations. 2010. Diversity and Unity in Federal Countries. A Global Dialogue on Federalism. Vol. 7. Montreal: Published for Forum of Federations and International Association of Centers for Federal Studies by McGill-Queen's University Press.

Mehretu, Assefa. 2012. "Ethnic Federalism and its Potential to Dismember the Ethiopian State." Progress and Development Studies 12 (2/3): 113-133.

Parker, Ben. "Ethiopian Constitution." University of Pennsylvania, African Studies Center. http://www.africa.upenn.edu/Hornet/Ethiopian_Constitution.html

Tewfik, Hashim. 2010. Transition to Federalism: The Ethiopian Experience. Ottawa: Forum of Federations.

Turton, David. 2006. Ethnic Federalism :The Ethiopian Experience in Comparative Perspective. Eastern African Studies. Oxford: James Currey. 
Zimmermann-Steinhart, Petra and Yacob Bekele. 2012. "The Implications of Federalism and Decentralisation on Socioeconomic Conditions in Ethiopia." Potchefstroom Electronic Law Journal 15 (2): 89-117.

\section{Annexe 1}

\section{Carte 1.0}

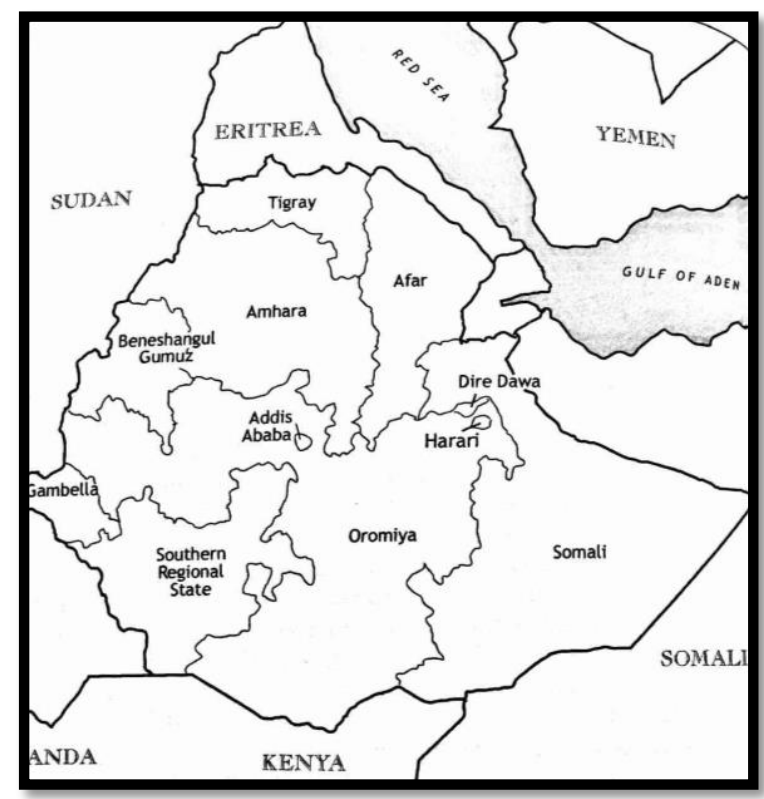

Source: Turton 2006, x

\section{Carte 1.1}

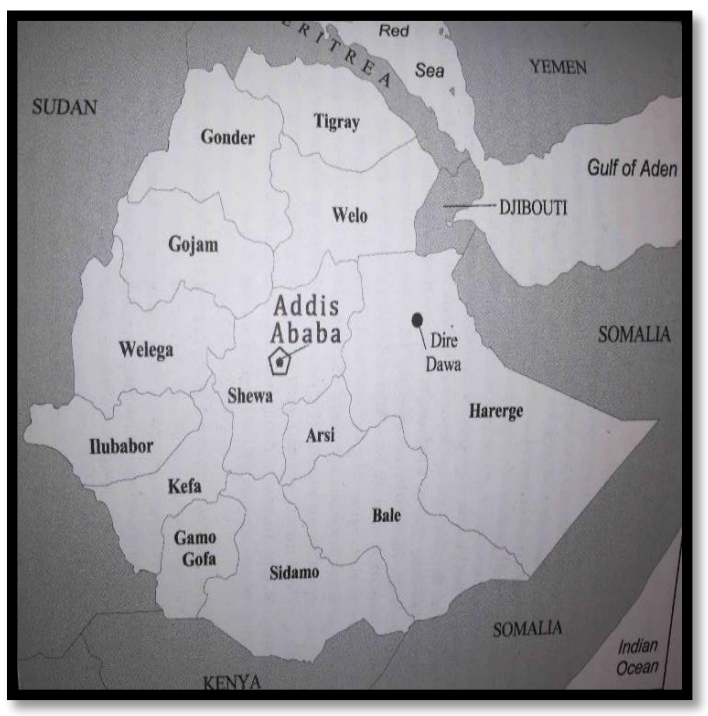

Source : Kincaid 2010,1 


\section{Annexe 2}

\section{Tableau 2.0}

Distribution of mother tongues (100 000+) and second languages in Ethiopia

\begin{tabular}{|c|c|c|c|c|}
\hline $\begin{array}{l}\text { Mother } \\
\text { tongue }\end{array}$ & Population & $\begin{array}{l}\text { Percentage } \\
\text { of total } \\
\text { population }\end{array}$ & $\begin{array}{l}\text { Second } \\
\text { language } \\
\text { population }\end{array}$ & $\begin{array}{l}\text { Percentag } \\
\text { of total } \\
\text { populatio }\end{array}$ \\
\hline Ambaric & $17,37,913$ & 32.70 & $5,104,150$ & 9.61 \\
\hline Oromiffa & $16,777,976$ & 31.58 & $1,535,434$ & 2.89 \\
\hline Tigrinya & $3,224,875$ & 6.07 & 146,933 & 0.28 \\
\hline Somali & $3,187,053$ & 6.00 & 95,572 & 0.18 \\
\hline Guragigna & $1,881,574$ & 3.54 & 208,358 & 0.39 \\
\hline Sidamigna & $1,876,329$ & 3.53 & 101,340 & 0.19 \\
\hline Welaitigna & $1,231,673$ & 2.32 & 89,801 & 0.17 \\
\hline Afarigna & 965,462 & 1.82 & 22,848 & 0.04 \\
\hline Hadiyigna & 923,958 & 1.74 & 150,889 & 0.28 \\
\hline Gamogna & 690,069 & 1.30 & 24,438 & 0.05 \\
\hline Gedeogna & 637,082 & 1.20 & 47,950 & 0.09 \\
\hline Keffigna & 569,626 & 1.07 & 46,720 & 0.09 \\
\hline Kembatigna & 487,655 & 0.92 & 68,607 & 0.13 \\
\hline Agew/ & 356,980 & 0.67 & 64,425 & 0.12 \\
\hline Awingiga & & & & \\
\hline Kulogna & 313,228 & 0.59 & 19,996 & 0.04 \\
\hline Goffigna & 233,340 & 0.44 & 33.449 & 0.06 \\
\hline Benchigna & 173,586 & 0.33 & 22,640 & 0.04 \\
\hline Arigna & 158.857 & 0.30 & 13,319 & 0.03 \\
\hline Konsogna & 149,508 & 0.28 & 5658 & 0.01 \\
\hline Agew///1/ & 143,369 & 0.27 & 11,026 & 0.02 \\
\hline Kamyrgna & The & 19 & & \\
\hline Alabigna & 126,257 & 0.24 & 25.271 & 0.05 \\
\hline Gumuzigna & 120.424 & 023 & 4379 & 0.01 \\
\hline Sebelawigna & 116,084 & 0.22 & 15,738 & 0.03 \\
\hline Koyrigna & 103,879 & 0.20 & 2371 & 000 \\
\hline
\end{tabular}

Source: Habtu 2004, 105 
Tableau 2.1

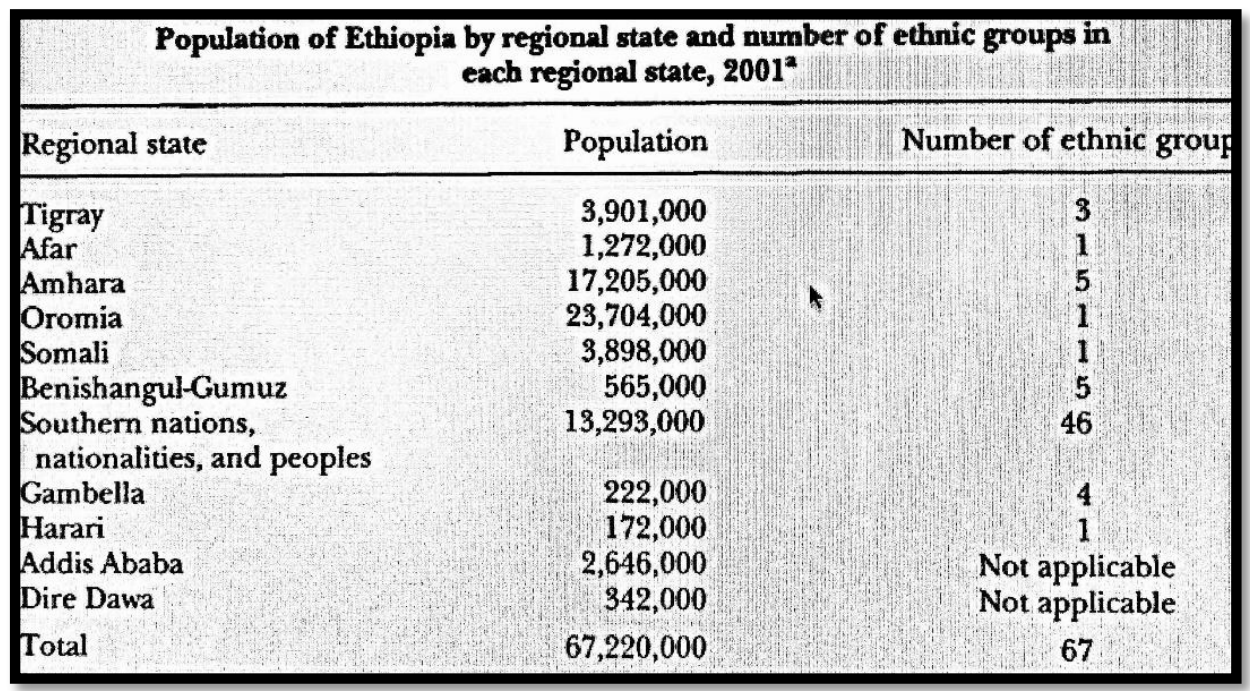

Source : Habtu 2005, 320

\section{Annexe 3}

Article 39 The Right of Nations, Nationalities and Peoples:

1. Every nation, nationality or people in Ethiopia shall have the unrestricted right to self determination up to secession.

2. Every nation, nationality and people shall have the right to speak, write and develop its language and to promote its culture, help it grow and flourish, and preserve its historical heritage.

3. Every nation, nationality or people in Ethiopia shall have the unrestricted right to administer itself; and this shall include the right to establish government institutions within the territory it inhabits and the right to fair representation in the federal and state governments.

4. The right to self determination up to secession of nation, nationality and peoples may be exercised:-

(a) where the demand for secession is approved by a two thirds (2/3rds) majority of the legislature of the nation, nationality or people concerned.

(b) where the Federal Government within three years upon receipt of the decision of the legislature of the nation, nationality or people demanding secession, organises a referendum for the nation, nationality or people demanding secession.

(c) where the demand for secession is supported by a simple majority vote in the referendum.

(d) where the Federal Government transfers power to the parliament of the nation, nationality or people which has opted for secession.

(e) where propert is partitioned in accordance with the law.

5. The term "nation, nationality and people" shall mean a community having the following characteristics: People having a common culture reflecting considerable uniformity or similarity of custom, a common language, belief in a common bond and identity, and a common consciousness the majority of whom live within a common territory. An alternative supported 
by a minority of the Council.

1. Every nationality in Ethiopia shall have the right to speak and write in its own language, and express, promote and develop it.

2. Every nationality in Ethiopia shall have the full right to administer itself .This right shall include the right to establish government institutions within the territory it inhabits and the right to fair representation in the federal and state governments.

3. Nationalities shall have, on the basis of the free choice of their peoples, the right to organise on a larger territory a self- administrative structure for running their internal affairs and establish governmental institutions for common self-administration.

4. Nationalities shall also have, on the basis of the free choice of their peoples, the right to establish regional self-administration, and such regional self-administrative unit shall be a member of the Federation.

5. For the purposes of this constitution, the term "nationality" shall mean a community having the following characteristics: people with a common culture reflecting considerable uniformity and a similarity of custom, a common language or (minority) languages of communication, a belief in a common bond and identity, the majority of whom live in a common territory.

6. Affiliated nationalities who share common characteristics but exhibiting varying cultures, common political and economic interests and believe in establishing, on the basis of the free choice of their peoples, a common administration may together decide to be recognized as a single nation or as one people.

7. In the event where the rights enumerated in this Article are violated, or by virtue of an unjust distribution of social wealth or an unfair distribution of the products of development, a sector of the population which has obtained recognition as a nation in accordance with Sub-Article (6) of this Article raises the demand for secession, the causes for which the demand was made shall be made to find solutions.

(a) any question arising out of the causes indicated herein above shall be submitted to the Constitutional Court for its consideration. Where the Constitutional Court subsequently finds that these causes reflect the views of the majority of the population, it may further investigate the causes and decide upon various solutions, including that of compensation or submit other recommendations for conciliation.

(b) The decision made, or the recommendation for conciliation made by the Constitutional Court shall be submitted to a joint meeting of the two Chambers of the Council, and the joint meeting may accept, reject or amend the decision or recommendation for conciliation.

(c) The decision passed by the joint meeting shall be submitted to the parliament of the selfadministration of the nation concerned.

(d) Where the parliament of the nation concerned rejects the joint meeting's decision, it may, by two-thirds majority vote submit an alternative recommendation for conciliation to the Council of the Federation or decide for a referendum on the secession issue to be conducted.

(e) The referendum shall be held three years after the nation's parliaments approval of the demand for secession in accordance with Sub-Article 1(d) of this Article.

(f) The decision for secession shall come into effect where it is supported by two-thirds majority vote of the population of the nation concerned.

Article 61 Council of the Federation: 
1. The Council of the Federation shall be composed of representatives of nations, nationalities and peoples of the member States of the Federation.

2. Each nation, nationality, people shall have at least one representative. Every additional one million people of the nation /nationality shall be represented by one additional representative. 3. The Members of the Council of the Federation may be elected by parliaments of the respective states. The parliaments may elect the representative either by themselves or through direct elections by the people.

Source: Parker,1994 